\title{
Persistence Property and Estimate on Momentum Support for the Integrable Degasperis-Procesi Equation
}

\author{
Zhengguang Guo ${ }^{1}$ and Liangbing Jin ${ }^{2}$ \\ ${ }^{1}$ College of Mathematics and Information Science, Wenzhou University, Wenzhou, Zhejiang 325035, China \\ ${ }^{2}$ Department of Mathematics, Zhejiang Normal University, Jinhua, Zhejiang 321004, China
}

Correspondence should be addressed to Zhengguang Guo; gzgmath@gmail.com

Received 24 April 2013; Accepted 4 October 2013

Academic Editor: T. Raja Sekhar

Copyright (c) 2013 Z. Guo and L. Jin. This is an open access article distributed under the Creative Commons Attribution License, which permits unrestricted use, distribution, and reproduction in any medium, provided the original work is properly cited.

It is shown that a strong solution of the Degasperis-Procesi equation possesses persistence property in the sense that the solution with algebraically decaying initial data and its spatial derivative must retain this property. Moreover, we give estimates of measure for the momentum support.

\section{Introduction}

Recently, Degasperis and Procesi [1] consider the following family of third order dispersive conservation laws:

$$
u_{t}+c_{0} u_{x}+\gamma u_{x x x}-\alpha^{2} u_{x x t}=\left(c_{1} u^{2}+c_{2} u_{x}^{2}+c_{3} u u_{x x}\right)_{x}
$$

where $\alpha, \gamma, c_{0}, c_{1}, c_{2}$, and $c_{3}$ are real constants. Within this family, only three equations that satisfy asymptotic integrability condition up to third order are singled out, namely, the KdV equation

$$
u_{t}+u_{x}+u u_{x}+u_{x x x}=0
$$

the Camassa-Holm equation

$$
u_{t}-u_{x x t}+3 u u_{x}=2 u_{x} u_{x x}+u u_{x x x}
$$

and a new equation (the Degasperis-Procesi equation, the DP equation, for simplicity) which can be written as (after rescaling) the dispersionless form [1]

$$
u_{t}-u_{x x t}+4 u u_{x}=3 u_{x} u_{x x}+u u_{x x x} .
$$

It is worth noting that in [2] both the Camassa-Holm and DP equations are derived as members of a one-parameter family of asymptotic shallow water approximations to the Euler equations: this is important because it shows that (after the addition of linear dispersion terms) both the CamassaHolm and DP equations are physically relevant; otherwise the DP equation would be of purely theoretical interest.

When $c_{1}=-3 c_{3} / 2 \alpha^{2}$ and $c_{2}=c_{3} / 2$ in (1), we recover the Camassa-Holm equation derived physically by Camassa and Holm in [3] by approximating directly the Hamiltonian for Euler's equations in the shallow water regime, where $u(x, t)$ represents the free surface above a flat bottom. There is also a geometric approach which is used to prove the least action principle holding for the Camassa-Holm equation, compared with [4]. It is worth pointing out that a fundamental aspect of the Camassa-Holm equation, the fact that it is a completely integrable system, was shown in $[5,6]$. Some satisfactory results have been obtained for this shallow water equation recently, we refer the readers to see [7-19].

Although, the DP equation (4) has a similar form to the Camassa-Holm equation and admits exact peakon solutions analogous to the Camassa-Holm peakons [20], these two equations are pretty different. The isospectral problem for equation (4) is

$$
\Psi_{x}-\Psi_{x x x}-\lambda y \Psi=0
$$

while for Camassa-Holm equation it is

$$
\Psi_{x x}-\frac{1}{4} \Psi-\lambda y \Psi=0
$$


where $y=u-u_{x x}$ for both cases. This implies that the inside structures of the DP equation (4) and the Camassa-Holm equation are truly different. However, we not only have some similar results [21-23], but also have considerable differences in the scattering/inverse scattering approach, compared with the discussion in $[5,6]$ and in the paper [24].

Analogous to the Camassa-Holm equation, (4) can be written in Hamiltonian form and has infinitely many conservation laws. Here we list some of the simplest conserved quantities [20]:

$$
\begin{aligned}
& H_{-1}=\int_{\mathbb{R}} u^{3} d x, \quad H_{0}=\int_{\mathbb{R}} y d x, \quad H_{1}=\int_{\mathbb{R}} y v d x, \\
& H_{5}=\int_{\mathbb{R}} y^{1 / 3} d x, \quad H_{7}=\int_{\mathbb{R}}\left(y_{x}^{2} y^{-7 / 3}+9 y^{-1 / 3}\right) d x,
\end{aligned}
$$

where $v=\left(4-\partial_{x}^{2}\right)^{-1} u$. So they are different from the invariants of the Camassa-Holm equation

$$
E(u)=\int_{\mathbb{R}}\left(u^{2}+u_{x}^{2}\right) d x, \quad F(u)=\int_{\mathbb{R}}\left(u^{3}+u u_{x}^{2}\right) d x .
$$

Set $Q=\left(1-\partial_{x}^{2}\right)$; then the operator $Q^{-1}$ in $\mathbb{R}$ can be expressed by

$$
Q^{-1} f=G * f=\frac{1}{2} \int_{\mathbb{R}} e^{-|x-y|} f(y) d y .
$$

Equation (4) can be written as

$$
u_{t}+u u_{x}+\partial_{x} G *\left(\frac{3}{2} u^{2}\right)=0
$$

while the Camassa-Holm equation can be written as

$$
u_{t}+u u_{x}+\partial_{x} G *\left(u^{2}+\frac{1}{2} u_{x}^{2}\right)=0 .
$$

On the other hand, the DP equation can also be expressed in the following momentum form:

$$
\begin{gathered}
y_{t}+y_{x} u=-3 y u_{x} \\
y=\left(1-\partial_{x}^{2}\right) u
\end{gathered}
$$

This formulation is important to motivate us to consider the measure of momentum support which is the second object of this paper, since we found that (12) is similar to the vorticity equation of the three-dimensional Euler equation for incompressible perfect fluids ( $U$ is the speed, and $\omega$ is its vorticity)

$$
\begin{aligned}
\omega_{t}+(U \cdot \nabla) \omega & =(\omega \cdot \nabla) U, \\
\operatorname{div} U & =0, \\
\operatorname{curl} U & =\omega .
\end{aligned}
$$

The stretching term $(\omega \cdot \nabla) U$ in (13) is similar to the term $-3 y u_{x}$ in (12).

One can follow the argument for the Camassa-Holm equation [8] to establish the following well posedness theorem for the Degasperis-Procesi equation.
Theorem 1 (see [23]). Given $u(x, t=0)=u_{0} \in H^{s}(\mathbb{R}), s>$ $3 / 2$, then there exist a $T$ and a unique solution $u$ to (4) (also (10)) such that

$$
u(x, t) \in C\left([0, T) ; H^{s}(\mathbb{R})\right) \cap C^{1}\left([0, T) ; H^{s-1}(\mathbb{R})\right) .
$$

It should be mentioned that due to the form of (10) (no derivative appears in the convolution term), Coclite and Karlsen [25] established global existence and uniqueness result for entropy weak solutions belonging to the class $L^{1}(\mathbb{R}) \cap B V(\mathbb{R})$.

\section{Unique Continuation}

The purpose of this section is to show that the solution to (10) and its first-order spatial derivative retain algebraic decay at infinity as their initial values do. Precisely, we prove.

Theorem 2. Assume that for some $T>0$ and $s>3 / 2, u \in$ $C\left([0, T] ; H^{s}(\mathbb{R})\right)$ is a strong solution of the initial value problem associated with (10), and that $u_{0}(x)=u(x, 0)$ satisfies that for some $\theta>1$

$$
\left|u_{0}(x)\right|, \quad\left|\partial_{x} u_{0}(x)\right|=O\left(x^{-\theta}\right) \text { as } x \uparrow \infty \text {. }
$$

Then

$$
|u(x, t)|, \quad\left|\partial_{x} u(x, t)\right|=O\left(x^{-\theta}\right) \quad \text { as } x \uparrow \infty,
$$

uniformly in the time interval $[0, T]$.

Notation. We will say that

$$
|f(x)|=O\left(x^{-\theta}\right) \quad \text { as } x \uparrow \infty \quad \text { if } \lim _{x \rightarrow \infty} \frac{|f(x)|}{x^{-\theta}}=L \text {, }
$$

where $L$ is a nonnegative constant.

Proof. We introduce the following notations:

$$
\begin{gathered}
F(u)=\frac{3}{2} u^{2}, \\
M=\sup _{t \in[0, T]}\|u(t)\|_{H^{s}} .
\end{gathered}
$$

Multiplying (10) by $u^{2 p-1}$ with $p \in Z^{+}$and integrating the result in the $x$-variable, one gets

$$
\int_{-\infty}^{\infty} u^{2 p-1}\left(u_{t}+u u_{x}+\partial_{x} G * F(u)\right) d x=0 .
$$

The first term in (20) is

$$
\begin{aligned}
\int_{-\infty}^{\infty} u^{2 p-1} u_{t} d x & =\int_{-\infty}^{\infty} \frac{1}{2 p} \frac{d u^{2 p}}{d t} d x \\
& =\frac{1}{2 p} \frac{d}{d t} \int_{-\infty}^{\infty} u^{2 p} d x=\|u(t)\|_{2 p}^{2 p-1} \frac{d}{d t}\|u(t)\|_{2 p}
\end{aligned}
$$


and for the rest, we have

$$
\begin{aligned}
\left|\int_{-\infty}^{\infty} u^{2 p-1} u u_{x} d x\right| & =\left|\int_{-\infty}^{\infty} u^{2 p} u_{x} d x\right| \\
& \leq\left\|u_{x}(t)\right\|_{\infty}\|u(t)\|_{2 p}^{2 p}, \\
\left|\int_{-\infty}^{\infty} u^{2 p-1} \partial_{x} G * F(u) d x\right| & \leq\|u(t)\|_{2 p}^{2 p-1}\left\|\partial_{x} G * F(u)(t)\right\|_{2 p} .
\end{aligned}
$$

From the above inequalities, we get

$$
\frac{d}{d t}\|u(t)\|_{2 p} \leq\left\|u_{x}(t)\right\|_{\infty}\|u(t)\|_{2 p}+\left\|\partial_{x} G * F(u)\right\|_{2 p} \text {, }
$$

and therefore, by Sobolev embedding theorem and Gronwall's inequality, there exists a constant $M$ such that

$$
\|u(t)\|_{2 p} \leq\left(\|u(0)\|_{2 p}+\int_{0}^{t}\left\|\partial_{x} G * F(u)\right\|_{2 p} d \tau\right) e^{M t}
$$

Since $f \in L^{1}(\mathbb{R}) \cap L^{\infty}(\mathbb{R})$ implies

$$
\lim _{q \rightarrow \infty}\|f\|_{q}=\|f\|_{\infty}
$$

taking the limits in (24) (note that $\partial_{x} G \in L^{1}$ and $F(u) \in L^{1} \cap$ $L^{\infty}$ ) from (25) we get

$$
\|u(t)\|_{\infty} \leq\left(\|u(0)\|_{\infty}+\int_{0}^{t}\left\|\partial_{x} G * F(u)\right\|_{\infty} d \tau\right) e^{M t}
$$

We will now repeat the above arguments using the barrier function

$$
\varphi_{N}(x)= \begin{cases}1, & x \leq 1 \\ x^{\theta}, & x \in(1, N) \\ N^{\theta}, & x \geq N\end{cases}
$$

where $N \in \mathbb{Z}^{+}$. Observe that for all $N$ we have

$$
0 \leq \varphi_{N}^{\prime}(x) \leq \theta \varphi_{N}(x) \quad \text { a.e. } x \in \mathbb{R}
$$

Using notation in (18), from (10) we obtain

$$
\left(u \varphi_{N}\right)_{t}+\left(u \varphi_{N}\right) u_{x}+\varphi_{N} \partial_{x} G * F(u)=0 .
$$

Hence, as in the weightless case (26), we get

$$
\begin{aligned}
\left\|u(t) \varphi_{N}\right\|_{\infty} \leq & e^{M t}\left\|u(0) \varphi_{N}\right\|_{\infty} \\
& +e^{M t} \int_{0}^{t}\left\|\varphi_{N} \partial_{x} G * F(u)\right\|_{\infty} d \tau .
\end{aligned}
$$

A simple calculation shows that there exists $C_{0}>0$ depending only on $\theta$ such that, for any $N \in \mathbb{Z}^{+}$,

$$
\frac{1}{2} \varphi_{N}(x) \int_{-\infty}^{\infty} e^{-|x-y|} \frac{1}{\varphi_{N}(y)} d y \leq C_{0} .
$$

Thus, for any appropriate function $f$ one finds that

$$
\begin{aligned}
& \left|\varphi_{N} \partial_{x} G * f^{2}(x)\right| \\
& \quad=\left|\frac{1}{2} \varphi_{N}(x) \int_{-\infty}^{\infty} \operatorname{sgn}(x-y) e^{-|x-y|} f^{2}(y) d y\right| \\
& \quad \leq \frac{\varphi_{N}(x)}{2} \int_{-\infty}^{\infty} e^{-|x-y|} \frac{1}{\varphi_{N}(y)} \varphi_{N}(y) f(y) f(y) d y \\
& \quad \leq\left(\frac{\varphi_{N}(x)}{2} \int_{-\infty}^{\infty} \frac{e^{-|x-y|}}{\varphi_{N}(y)} d y\right)\left\|\varphi_{N} f\right\|_{\infty}\|f\|_{\infty} \\
& \quad \leq C_{0}\left\|\varphi_{N} f\right\|_{\infty}\|f\|_{\infty} .
\end{aligned}
$$

Combining with (30), we get

$$
\left\|u(t) \varphi_{N}\right\|_{\infty} \leq C_{1}\left(\left\|u_{0} \varphi_{N}\right\|_{\infty}+\int_{0}^{t}\left\|\varphi_{N} u\right\|_{\infty} d \tau\right)
$$

where $C_{1}=C_{1}(M ; T)>$,0 . By Gronwall's inequality, there exists a constant $\widetilde{C}$ for any $t \in[0, T]$ such that

$$
\left\|\varphi_{N} u\right\|_{\infty} \leq \widetilde{C}\left\|u_{0} \varphi_{N}\right\|_{\infty} \leq \widetilde{C}\left\|u_{0} \cdot \max \left(1, x^{\theta}\right)\right\|_{\infty} .
$$

Finally, taking the limit as $N$ goes to infinity in (34) we find that for any $t \in[0, T]$

$$
\left|u(x, t) x^{\theta}\right| \leq \widetilde{C}\left\|u_{0} \cdot \max \left(1, x^{\theta}\right)\right\|_{\infty} .
$$

From (15), we get $|u(x, t)|=O\left(x^{-\theta}\right)$ as $x \uparrow \infty$.

Next, differentiating (10) in the $x$-variable produces the equation

$$
u_{x t}+u u_{x x}+u_{x}^{2}+\partial_{x}^{2} G *\left(\frac{3}{2} u^{2}\right)=0
$$

Again, multiplying (36) by $u_{x}^{2 p-1},\left(p \in \mathbb{Z}^{+}\right)$, integrating the result in the $x$-variable, and using integration by parts

$$
\begin{aligned}
\int_{-\infty}^{\infty} u u_{x x}\left(u_{x}\right)^{2 p-1} d x & =\int_{-\infty}^{\infty} u \frac{\left(u_{x}\right)^{2 p}}{2 p} d x \\
& =-\frac{1}{2 p} \int_{-\infty}^{\infty} u_{x}\left(u_{x}\right)^{2 p} d x
\end{aligned}
$$

one gets the inequality

$$
\frac{d}{d t}\left\|u_{x}(t)\right\|_{2 p} \leq 2\left\|u_{x}(t)\right\|_{\infty}\left\|u_{x}(t)\right\|_{2 p}+\left\|\partial_{x}^{2} G * F(u)\right\|_{2 p},
$$

and therefore as before

$$
\left\|u_{x}(t)\right\|_{2 p} \leq\left(\left\|u_{x}(0)\right\|_{2 \mathrm{p}}+\int_{0}^{t}\left\|\partial_{x}^{2} G * F(u)\right\|_{2 p} d \tau\right) e^{2 M t} .
$$


Since $\partial_{x}^{2} G=G-\delta$, we can use (25) and pass to the limit in (39) to obtain

$$
\left\|u_{x}(t)\right\|_{\infty} \leq\left(\left\|u_{x}(0)\right\|_{\infty}+\int_{0}^{t}\left\|\partial_{x}^{2} G * F(u)\right\|_{\infty} d \tau\right) e^{2 M t}
$$

from (36) we get

$$
\partial_{t}\left(u_{x} \varphi_{N}\right)+u u_{x x} \varphi_{N}+\left(u_{x} \varphi_{N}\right) u_{x}+\varphi_{N} \partial_{x}^{2} G * F(u)=0 .
$$

We need to eliminate the second derivatives in the second term in (41). Thus, combining integration by parts and (28), we find

$$
\begin{aligned}
\mid \int_{-\infty}^{\infty} & u u_{x x} \varphi_{N}\left(u_{x} \varphi_{N}\right)^{2 p-1} d x \mid \\
\quad & \left|\int_{-\infty}^{\infty} u\left(u_{x} \varphi_{N}\right)^{2 p-1}\left(\partial_{x}\left(u_{x} \varphi_{N}\right)-u_{x} \varphi_{N}^{\prime}\right) d x\right| \\
& =\left|\int_{-\infty}^{\infty} u\left(\partial_{x}\left(\frac{\left(u_{x} \varphi_{N}\right)^{2 p}}{2 p}\right)-u_{x} \varphi_{N}^{\prime}\left(u_{x} \varphi_{N}\right)^{2 p-1}\right) d x\right| \\
& \leq \kappa \cdot\left(\|u(t)\|_{\infty}+\left\|\partial_{x} u(t)\right\|_{\infty}\right)\left\|\partial_{x} u \varphi_{N}\right\|_{2 p}^{2 p}
\end{aligned}
$$

Since $\partial_{x}^{2} G=G-\delta$, the argument in (32) also shows that

$$
\left|\varphi_{N} \partial_{x}^{2} G * f^{2}(x)\right| \leq C_{0}\left\|\varphi_{N} f\right\|_{\infty}\|f\|_{\infty} .
$$

Similarly, we get

$$
\begin{aligned}
& \left\|u_{x}(t) \varphi_{N}\right\|_{\infty} \\
& \quad \leq C_{2}\left(\left\|u_{x}(0) \varphi_{N}\right\|_{\infty}+\int_{0}^{t}\left\|u(\tau) \varphi_{N}\right\|_{\infty} d \tau\right),
\end{aligned}
$$

where $C_{2}=C_{2}(M ; T)$.

Then, taking the limit as $N$ goes to infinity, we find that for any $t \in[0, T]$

$$
\left|u_{x}(t) x^{\theta}\right| \leq C_{2}\left(\left\|u_{x}(0) x^{\theta}\right\|_{\infty}+\int_{0}^{t}\left\|u(\tau) x^{\theta}\right\|_{\infty} d \tau\right) .
$$

Since $|u(x, t)|=O\left(x^{-\theta}\right)$ as $x \uparrow \infty$ and (15), we get

$$
\left|\partial_{x} u(x, t)\right|=O\left(x^{-\theta}\right), \quad \text { as } x \uparrow \infty .
$$

This completes the proof.

\section{Measure of Momentum Support}

It is known that, for the Degasperis-Procesi equation, the momentum density $y(x, t)$ with compactly supported initial data $y_{0}(x)$ will retain this property; that is, $y(x, t)$ is also compactly supported [21]. However, the same argument for $u(x, t)$ is false [21]. Note that a detailed description of solution $u(x, t)$ outside of the support of $y(x, t)$ is given in [26, 27].
Moreover, the exponential behavior of $u$ in $x$ outside this support is obvious. The comparison of the DP equation and the incompressible Euler equation above implies that the momentum $y(x, t)$ in (12) plays a similar role as the vorticity does in (13). This motivates us to estimate the size of supp $y(t, \cdot)$ for strong solutions. The approach is inspired by the work of Kim [28] and the recent work [29].

We first introduce the particle trajectory method. Let $u \in$ $C\left([0, T], H^{3}(\mathbb{R})\right) \cap C^{1}\left([0, T], H^{2}(\mathbb{R})\right)$ be a strong solution of (4) guaranteed by the well posedness Theorem 1 . Let $s \in$ $[0, T], q(t ; \alpha, s)$ be the solution of the following initial value problem:

$$
\begin{gathered}
\frac{d q(t ; \alpha, s)}{d t}=u(s+t, q(t ; \alpha, s)), s, s+t \in[0, T], \quad \alpha \in \mathbb{R}, \\
q(0 ; \alpha, s)=\alpha, \quad \alpha \in \mathbb{R} .
\end{gathered}
$$

Then, $q(t ; \cdot, s): \mathbb{R} \rightarrow \mathbb{R}$ is an increasing diffeomorphism. It is shown $[21,23]$ that

$$
y(q(t ; x, 0), t) q_{x}^{3}(t ; x, 0)=y(x, 0) ;
$$

this implies that the support of $y$ propagates along the flow. Set $D(t)$ to be the support of $y(\cdot, t)$. Let $\psi \in L^{2}(D(s))$, and let $\psi^{t} \in L^{2}(D(s+t))$ be given by the following:

$$
\psi^{t}(q(t ; \alpha, s))=\psi(\alpha) .
$$

Moreover, we also want to mention the standard argument on the first Dirichlet eigenvalue problem. Let $\Omega$ be an open interval in $\mathbb{R}$, and, $\lambda_{1}(\Omega)$ be the first Dirichlet eigenvalue of the Laplacian on $\Omega$. Then we have

$$
\lambda_{1}(\Omega)=\inf \left\{\left\|\phi^{\prime}\right\|_{L^{2}(\Omega)}^{2} \mid \phi \in H_{0}^{1}(\Omega) \text { with }\|\phi\|_{L^{2}(\Omega)}=1\right\} .
$$

It is just $(\pi /|\Omega|)^{2}$ and the normalized eigenfunctions are the suitable translations of

$$
\pm\left(\frac{2}{|\Omega|}\right)^{1 / 2} \sin \left(\frac{\pi x}{|\Omega|}\right) .
$$

Theorem 3. Let $y \in C\left([0, T] ; H^{1}(\mathbb{R})\right) \cap C^{1}\left([0, T] ; L^{2}(\mathbb{R})\right)$ be a strong solution of $(12)$. Let $D(t)$ be the support of $y(\cdot, t)$ for $t \in[0, T]$ with its initial $D(0)$ being connected.

(I) Suppose there exists a positive constant $K$ such that $u_{x}(x, k)>-K$ for $(x, t) \in \mathbb{R} \times[0, T]$. Then

$$
\begin{aligned}
& |D(0)| e^{-\left(\exp (5 K T / 2)\left\|y_{0}\right\|_{L^{2}(\mathbb{R})}\right) t} \\
& \quad \leq|D(t)| \leq|D(0)| e^{\left(\exp (5 K T / 2)\left\|y_{0}\right\|_{L^{2}(\mathbb{R})}\right) t} .
\end{aligned}
$$

(II) $y_{0}$ does not change sign or

$$
\begin{aligned}
& y_{0}(x) \leq 0, \quad x \in\left(-\infty, x_{0}\right), \\
& y_{0}(x) \geq 0, \quad x \in\left(x_{0}, \infty\right),
\end{aligned}
$$


and $y_{0} \in H^{1}(\mathbb{R}) \cap L^{1}(\mathbb{R})$; then, for all $t \geq 0$

$$
\begin{aligned}
|D(0)| e^{-\left\|y_{0}\right\|_{L^{1}(\mathbb{R})^{t}}} & \leq|D(t)| \\
& \leq|D(0)| e^{\left\|y_{0}\right\|_{L^{1}(\mathbb{R})^{t}}} .
\end{aligned}
$$

Proof. (I) The relation of momenta $y$ and $u$ gives

$$
\begin{gathered}
u(x, t)=\frac{1}{2} \int_{\mathbb{R}} e^{-|x-\xi|} y(\xi, t) d \xi, \\
u_{x}(x, t)=\frac{1}{2} \int_{\mathbb{R}} \operatorname{sgn}(\xi-x) e^{-|x-\xi|} y(\xi, t) d \xi .
\end{gathered}
$$

Then, we have by (12) and the lower bound of $u_{x}$

$$
\begin{aligned}
& \frac{d}{d t} \int_{\mathbb{R}} y^{2}(x, t) d x \\
& \quad=-5 \int_{\mathbb{R}} u_{x}(x, t) y^{2}(x, t) d x \leq 5 K \int_{\mathbb{R}} y^{2}(x, t) d x
\end{aligned}
$$

Thus

$$
\frac{d}{d t}\|y(x, t)\|_{L^{2}}^{2} \leq 5 K\|y(x, t)\|_{L^{2}}^{2}
$$

Therefore, (56), (58), and Gronwall inequality imply that

$$
\left|u_{x}(x, t)\right| \leq \frac{1}{2}\|y(x, t)\|_{L^{2}} \leq \frac{1}{2} e^{5 K T / 2}\left\|y_{0}\right\|_{L^{2}} .
$$

On the other hand, due to Propositions A.2 and A.3, $\lambda_{1}(D(s))$ is Lipschitz and differentiable almost everywhere. Moreover, we have

$$
-4 M_{1} \lambda_{1}(D(s)) \leq \frac{d}{d s} \lambda_{1}(D(s)) \leq 4 M_{1} \lambda_{1}(D(s)) .
$$

Then, it follows that

$$
e^{-4 M_{1} s} \lambda_{1}(D(0)) \leq \lambda_{1}(D(s)) \leq e^{4 M_{1} s} \lambda_{1}(D(0))
$$

with $\lambda_{1}(D(s))=\pi^{2} /|D(s)|^{2}$. So (52) follows from (61) and (59).

(II) If $y_{0} \in H^{1}(\mathbb{R}) \cap L^{1}(\mathbb{R})$ does not change sign, we conclude that solutions of (10) exist globally in time. Equality (56) and the conservation of $\int_{\mathbb{R}} y(x, t) d x$ yield

$$
\left|u_{x}(x, t)\right| \leq \frac{1}{2}\|y(x, t)\|_{L^{1}(\mathbb{R})}=\frac{1}{2}\left\|y_{0}(x)\right\|_{L^{1}(\mathbb{R})} .
$$

By similar arguments of (I), constant $M_{1}$ in (61) can be replaced by $\left\|y_{0}(x)\right\|_{L^{1}(\mathbb{R})} / 2$; then (54) follows. If (53) is satisfied, we know that the solution of (10) exists globally in time $[21,30]$. From (53) and (48), it is easy to get

$$
\begin{aligned}
& y(x, t) \leq 0, \quad x \in\left(-\infty, q\left(x_{0}, t\right)\right), \\
& y(x, t) \geq 0, \quad x \in\left(q\left(x_{0}, t\right), \infty\right),
\end{aligned}
$$

where we denote $q(t ; x, s)$ with $s=0$ by $q(x, t)$. By direct computation, we have

$$
\int_{\mathbb{R}}|y(x, t)| d x=\int_{q\left(x_{0}, t\right)}^{\infty} y(x, t) d x-\int_{-\infty}^{q\left(x_{0}, t\right)} y(x, t) d x .
$$

Next, we prove that $\|y(x, t)\|_{L^{1}(\mathbb{R})}$ is decreasing with respect to time. To this end, one gets, by differentiating (64) with respect to $t$ and integrating by parts,

$$
\begin{aligned}
\frac{d}{d t} \int_{\mathbb{R}}|y(x, t)| d x= & \int_{q\left(x_{0}, t\right)}^{\infty} y_{t}(x, t) d x \\
& -\int_{-\infty}^{q\left(x_{0}, t\right)} y_{t}(x, t) d x \\
& -2(y u)\left(q\left(x_{0}, t\right), t\right) \\
= & -\int_{q\left(x_{0}, t\right)}^{\infty}\left(y_{x} u+3 y u_{x}\right) d x \\
& +\int_{-\infty}^{q\left(x_{0}, t\right)}\left(y_{x} u+3 y u_{x}\right) d x \\
& -2(y u)\left(q\left(x_{0}, t\right), t\right) \\
= & -2 \int_{q\left(x_{0}, t\right)}^{\infty} y u_{x} d x+2 \int_{-\infty}^{q\left(x_{0}, t\right)} y u_{x} d x \\
= & u^{2}\left(q\left(x_{0}, t\right), t\right)-u_{x}^{2}\left(q\left(x_{0}, t\right), t\right) \\
= & \int_{q\left(x_{0}, t\right)}^{\infty} e^{-\xi} y(\xi, t) d x \int_{-\infty}^{q\left(x_{0}, t\right)} e^{\xi} y(\xi, t) d x \\
\leq & 0 .
\end{aligned}
$$

This implies that

$$
\left|u_{x}(x, t)\right| \leq \frac{1}{2}\|y(x, t)\|_{L^{1}(\mathbb{R})} \leq \frac{1}{2}\left\|y_{0}(x)\right\|_{L^{1}(\mathbb{R})} .
$$

Therefore, (54) follows by replacing $M_{1}$ with $\left\|y_{0}(x)\right\|_{L^{1}(\mathbb{R})} / 2$ in (61).

\section{Appendix}

The following propositions with standard proofs are known in [29]; we list them here only for convenience of readers.

Proposition A.1. Let $s, s+t \in[0, T], \alpha \in D(s)$, and $\psi \in$ $H_{0}^{1}(D(s)) ; u_{x}$ can be bounded by a constant $M_{1}$; then

(a)

$$
e^{-M_{1}|t|} \leq q_{\alpha}(t ; \alpha, s) \leq e^{M_{1}|t|}
$$

(b)

$$
\begin{aligned}
\left|\psi^{\prime}(\alpha)\right| e^{-M_{1}|t|} & \leq\left|\left(\psi^{t}\right)^{\prime}(q(t ; \alpha, s))\right| \\
& \leq\left|\psi^{\prime}(\alpha)\right| e^{M_{1}|t|}
\end{aligned}
$$


(c)

$$
\begin{aligned}
\|\psi\|_{L^{2}(D(s))} e^{-M_{1}|t| / 2} & \leq\left\|\psi^{t}\right\|_{L^{2}(D(s+t))} \\
& \leq\|\psi\|_{L^{2}(D(s))} e^{M_{1}|t| / 2} .
\end{aligned}
$$

Proof. (a) Differentiating (47) with respect to $\alpha$, we obtain

$$
\frac{d q_{t}}{d \alpha}=u_{q} q_{\alpha}
$$

Since $q(t ; \cdot, s): \mathbb{R} \rightarrow \mathbb{R}$ is an increasing diffeomorphism, then $q_{\alpha}>0$. Combining the bound of $u_{x}$, there holds

$$
-M_{1} q_{\alpha} \leq q_{\alpha t} \leq M_{1} q_{\alpha}
$$

This can be solved as (a).

(b) Differentiating (49) with respect to $\alpha$ to get

$$
\psi_{q}^{t} q_{\alpha}=\psi^{\prime}(\alpha)
$$

then (A.2) is a direct consequence of (A.1). that

(c) Equation (49) and the definition of Sobolev norm give

$$
\left\|\psi^{t}\right\|_{L^{2}(D(s+t))}^{2}=\int_{D(s+t)} \psi^{t}(x)^{2} d x=\int_{D(s)} \psi^{2}(\alpha) q_{\alpha} d \alpha,
$$

where we have used the change of variable $x=q(t ; \alpha, s)$. So (A.3) follows from (A.1).

Proposition A.2. Under the hypothesis of Theorem 3, for s, $s+$ $t \in[0, T]$,

$$
\begin{aligned}
& \lim _{t \rightarrow 0^{+}} \sup \frac{\lambda_{1}(D(s+t))-\lambda_{1}(D(s))}{t} \leq 4 M_{1} \lambda_{1}(D(s)), \\
& \lim _{t \rightarrow 0^{-}} \inf \frac{\lambda_{1}(D(s+t))-\lambda_{1}(D(s))}{t} \geq-4 M_{1} \lambda_{1}(D(s)) .
\end{aligned}
$$

Proof. Let $t>0, \phi_{1} \in H_{0}^{1}(D(s))$ with $\left\|\phi_{1}\right\|_{L^{2}(D(s))}=1$ be a first normalized eigenfunction on $D(s)$. Then, for $\varphi \in H_{0}^{1}(D(s+t))$ with $\|\varphi\|_{L^{2}(D(s+t))}=1$, we have

$$
\begin{aligned}
\lambda_{1}(D(s+t))-\lambda_{1}(D(s))= & \inf \left\|\varphi^{\prime}\right\|_{L^{2}(D(s+t))}^{2}-\left\|\phi_{1}^{\prime}\right\|_{L^{2}(D(s))}^{2} \\
\leq & \left\|\phi_{1}^{t}\right\|_{L^{2}(D(s+t))}^{-2}\left\|\left(\phi_{1}^{t}\right)^{\prime}\right\|_{L^{2}(D(s+t))}^{2} \\
& -\left\|\phi_{1}^{\prime}\right\|_{L^{2}(D(s))}^{2}
\end{aligned}
$$

Furthermore

$$
\begin{aligned}
& \left\|\phi_{1}^{t}\right\|_{L^{2}(D(s+t))}^{-2}\left\|\left(\phi_{1}^{t}\right)^{\prime}\right\|_{L^{2}(D(s+t))}^{2} \\
& \quad=\left\|\phi_{1}^{t}\right\|_{L^{2}(D(s+t))}^{-2} \int_{D(s)}\left[\left(\phi_{1}^{t}\right)^{\prime}\right]^{2} q_{\alpha} d \alpha \\
& \leq\left\|\phi_{1}^{t}\right\|_{L^{2}(D(s+t))}^{-2} e^{3 M_{1} t}\left\|\phi_{1}^{\prime}\right\|_{L^{2}(D(s))}^{2} \\
& \leq e^{4 M_{1} t}\left\|\phi_{1}^{\prime}\right\|_{L^{2}(D(s))}^{2} .
\end{aligned}
$$

Combing (A.9) and (A.10) together yields

$$
\begin{aligned}
\lim _{t \rightarrow 0^{+}} & \sup \frac{\lambda_{1}(D(s+t))-\lambda_{1}(D(s))}{t} \\
& \leq \lim _{t \rightarrow 0^{+}} \sup \frac{e^{4 M_{1} t}\left\|\phi_{1}^{\prime}\right\|_{L^{2}(D(s))}^{2}-\left\|\phi_{1}^{\prime}\right\|_{L^{2}(D(s))}^{2}}{t} \\
& =4 M_{1} \lambda_{1}(D(s)) .
\end{aligned}
$$

The second one follows by similar arguments for $t<0$.

Proposition A.3. Under the hypothesis of Theorem 3, for s, $s+$ $t \in[0, T]$

$$
\begin{aligned}
& \lim _{t \rightarrow 0^{-}} \sup \frac{\lambda_{1}(D(s+t))-\lambda_{1}(D(s))}{t} \leq 4 M_{1} \lambda_{1}(D(s)), \\
& \lim _{t \rightarrow 0^{+}} \inf \frac{\lambda_{1}(D(s+t))-\lambda_{1}(D(s))}{t} \geq-4 M_{1} \lambda_{1}(D(s)) .
\end{aligned}
$$

Proof. Let $\phi_{1} \in H_{0}^{1}(D(s))$ with $\left\|\phi_{1}\right\|_{L^{2}(D(s))}=1$ be a first normalized eigenfunction on $D(s)$, and let $\phi_{2} \in L^{2}(D(s))$ be such that its $t$-transport is a normalized first eigenfunction on $D(s+t)$. For $t>0$, using the left halves of (A.1) and (A.2) and then the right half of (A.3) we get

$$
\begin{aligned}
\left\|\left(\phi_{2}^{t}\right)^{\prime}\right\|_{L^{2}(D(s+t))}^{2} & =\int_{D(s+t)}\left[\left(\phi_{2}^{t}(x)\right)^{\prime}\right]^{2} d x \\
& =\int_{D(s)}\left[\left(\phi_{2}^{t}\right)^{\prime}\right]^{2} q_{\alpha} d \alpha \\
& \geq e^{-3 M_{1} t} \int_{D(s)}\left[\phi_{2}^{\prime}(\alpha)\right]^{2} d \alpha \\
& =e^{-3 M_{1} t}\left\|\phi_{2}\right\|_{L^{2}(D(s))}^{2}\left\|\left(\frac{\phi_{2}}{\left\|\phi_{2}\right\|_{L^{2}(D(s))}^{2}}\right)^{\prime}\right\|_{L^{2}(D(s))}^{2} \\
& \geq e^{-4 M_{1} t}\left\|\phi_{2}^{t}\right\|_{L^{2}(D(s+t))}^{2} \lambda_{1}(D(s)) \\
& =e^{-4 M_{1} t} \lambda_{1}(D(s)) .
\end{aligned}
$$

Hence

$$
\begin{aligned}
\lim _{t \rightarrow 0^{+}} & \inf \frac{\lambda_{1}(D(s+t))-\lambda_{1}(D(s))}{t} \\
& \geq \lim _{t \rightarrow 0^{+}} \inf \frac{e^{-4 M_{1} t}-1}{t} \lambda_{1}(D(s)) \\
& =-4 M_{1} \lambda_{1}(D(s)) .
\end{aligned}
$$

The other part is similar.

\section{Acknowledgments}

This work was partially supported by ZJNSF, under Grant nos. LQ12A01009 and LQ13A010008, and NSFC, under Grant nos. 11301394,11226176, and 11226172 . 


\section{References}

[1] A. Degasperis and M. Procesi, "Asymptotic integrability," in Symmetry and Perturbation Theory, pp. 23-37, World Science Publisher, River Edge, NJ, USA, 1999.

[2] H. R. Dullin, G. A. Gottwald, and D. D. Holm, "Camassa-Holm, Korteweg-de Vries-5 and other asymptotically equivalent equations for shallow water waves," Fluid Dynamics Research, vol. 33, no. 1-2, pp. 73-95, 2003.

[3] R. Camassa and D. D. Holm, "An integrable shallow water equation with peaked solitons," Physical Review Letters, vol. 71, no. 11, pp. 1661-1664, 1993.

[4] A. Constantin and B. Kolev, "On the geometric approach to the motion of inertial mechanical systems," Journal of Physics A, vol. 35, no. 32, pp. R51-R79, 2002.

[5] A. Constantin, "On the inverse spectral problem for the Camassa-Holm equation," Journal of Functional Analysis, vol. 155, no. 2, pp. 352-363, 1998.

[6] A. Constantin and H. P. McKean, "A shallow water equation on the circle," Communications on Pure and Applied Mathematics, vol. 52, no. 8, pp. 949-982, 1999.

[7] A. Bressan and A. Constantin, "Global conservative solutions of the Camassa-Holm equation," Archive for Rational Mechanics and Analysis, vol. 183, no. 2, pp. 215-239, 2007.

[8] A. Constantin and J. Escher, "Well-posedness, global existence, and blowup phenomena for a periodic quasi-linear hyperbolic equation," Communications on Pure and Applied Mathematics, vol. 51, no. 5, pp. 475-504, 1998.

[9] A. Constantin and W. A. Strauss, "Stability of the CamassaHolm solitons," Journal of Nonlinear Science, vol. 12, no. 4, pp. 415-422, 2002.

[10] Z. Guo, "Blow up, global existence, and infinite propagation speed for the weakly dissipative Camassa-Holm equation," Journal of Mathematical Physics, vol. 49, no. 3, Article ID 033516, 2008.

[11] A. A. Himonas, G. Misiołek, G. Ponce, and Y. Zhou, "Persistence properties and unique continuation of solutions of the Camassa-Holm equation," Communications in Mathematical Physics, vol. 271, no. 2, pp. 511-522, 2007.

[12] Y. A. Li and P. J. Olver, "Well-posedness and blow-up solutions for an integrable nonlinearly dispersive model wave equation," Journal of Differential Equations, vol. 162, no. 1, pp. 27-63, 2000.

[13] H. P. McKean, "Breakdown of a shallow water equation," The Asian Journal of Mathematics, vol. 2, no. 4, pp. 867-874, 1998.

[14] H. P. McKean, "Breakdown of the Camassa-Holm equation," Communications on Pure and Applied Mathematics, vol. 57, no. 3, pp. 416-418, 2004.

[15] G. Misiołek, "Classical solutions of the periodic Camassa-Holm equation," Geometric and Functional Analysis, vol. 12, no. 5, pp. 1080-1104, 2002.

[16] S. Shkoller, "Geometry and curvature of diffeomorphism groups with $H^{1}$ metric and mean hydrodynamics," Journal of Functional Analysis, vol. 160, no. 1, pp. 337-365, 1998.

[17] Z. Xin and P. Zhang, "On the weak solutions to a shallow water equation," Communications on Pure and Applied Mathematics, vol. 53, no. 11, pp. 1411-1433, 2000.

[18] Z. Yin, "On the Cauchy problem for an integrable equation with peakon solutions," Illinois Journal of Mathematics, vol. 47, no. 3 , pp. 649-666, 2003.

[19] Y. Zhou, "Wave breaking for a periodic shallow water equation," Journal of Mathematical Analysis and Applications, vol. 290, no. 2, pp. 591-604, 2004.
[20] A. Degasperis, D. D. Holm, and A. N. I. Hone, "A new integrable equation with peakon solutions," Theoretical and Mathematical Physics, vol. 133, no. 2, pp. 1463-1474, 2002.

[21] Z. Guo, "Some properties of solutions to the weakly dissipative Degasperis-Procesi equation," Journal of Differential Equations, vol. 246, no. 11, pp. 4332-4344, 2009.

[22] D. Henry, "Persistence properties for the Degasperis-Procesi equation," Journal of Hyperbolic Differential Equations, vol. 5, no. 1, pp. 99-111, 2008.

[23] Y. Zhou, "Blow-up phenomenon for the integrable DegasperisProcesi equation," Physics Letters. A, vol. 328, no. 2-3, pp. 157$162,2004$.

[24] A. Constantin, "On the scattering problem for the CamassaHolm equation," Proceedings of the Royal Society London A, vol. 457, no. 2008, pp. 953-970, 2001.

[25] G. M. Coclite and K. H. Karlsen, "On the well-posedness of the Degasperis-Procesi equation," Journal of Functional Analysis, vol. 233, no. 1, pp. 60-91, 2006.

[26] D. Henry, "Infinite propagation speed for the DegasperisProcesi equation," Journal of Mathematical Analysis and Applications, vol. 311, no. 2, pp. 755-759, 2005.

[27] O. G. Mustafa, "A note on the Degasperis-Procesi equation," Journal of Nonlinear Mathematical Physics, vol. 12, no. 1, pp. 1014, 2005.

[28] N. Kim, "Eigenvalues associated with the vortex patch in 2-D Euler equations," Mathematische Annalen, vol. 330, no. 4, pp. 747-758, 2004.

[29] S.-G. Kang and T.-M. Tang, "The support of the momentum density of the Camassa-Holm equation," Applied Mathematics Letters, vol. 24, no. 12, pp. 2128-2132, 2011.

[30] Y. Liu and Z. Yin, "Global existence and blow-up phenomena for the Degasperis-Procesi equation," Communications in Mathematical Physics, vol. 267, no. 3, pp. 801-820, 2006. 


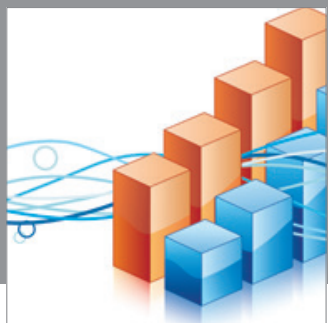

Advances in

Operations Research

mansans

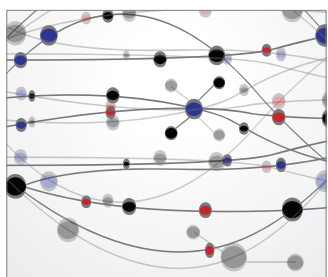

The Scientific World Journal
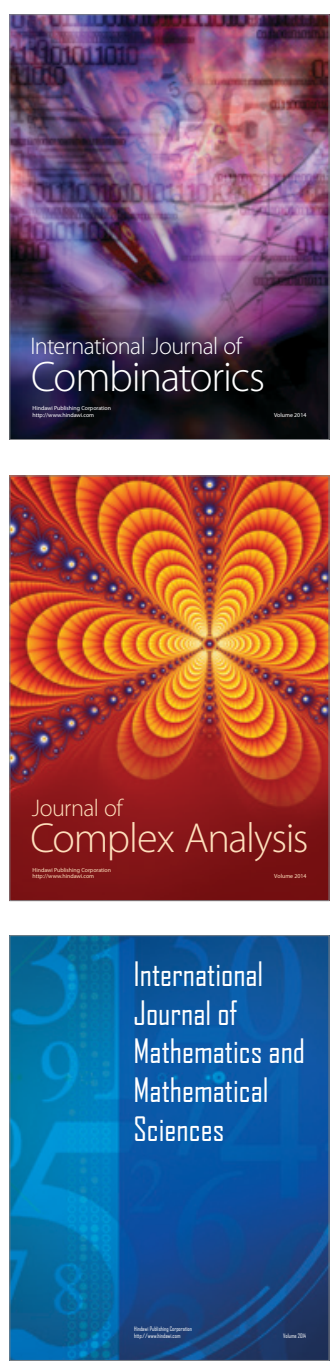
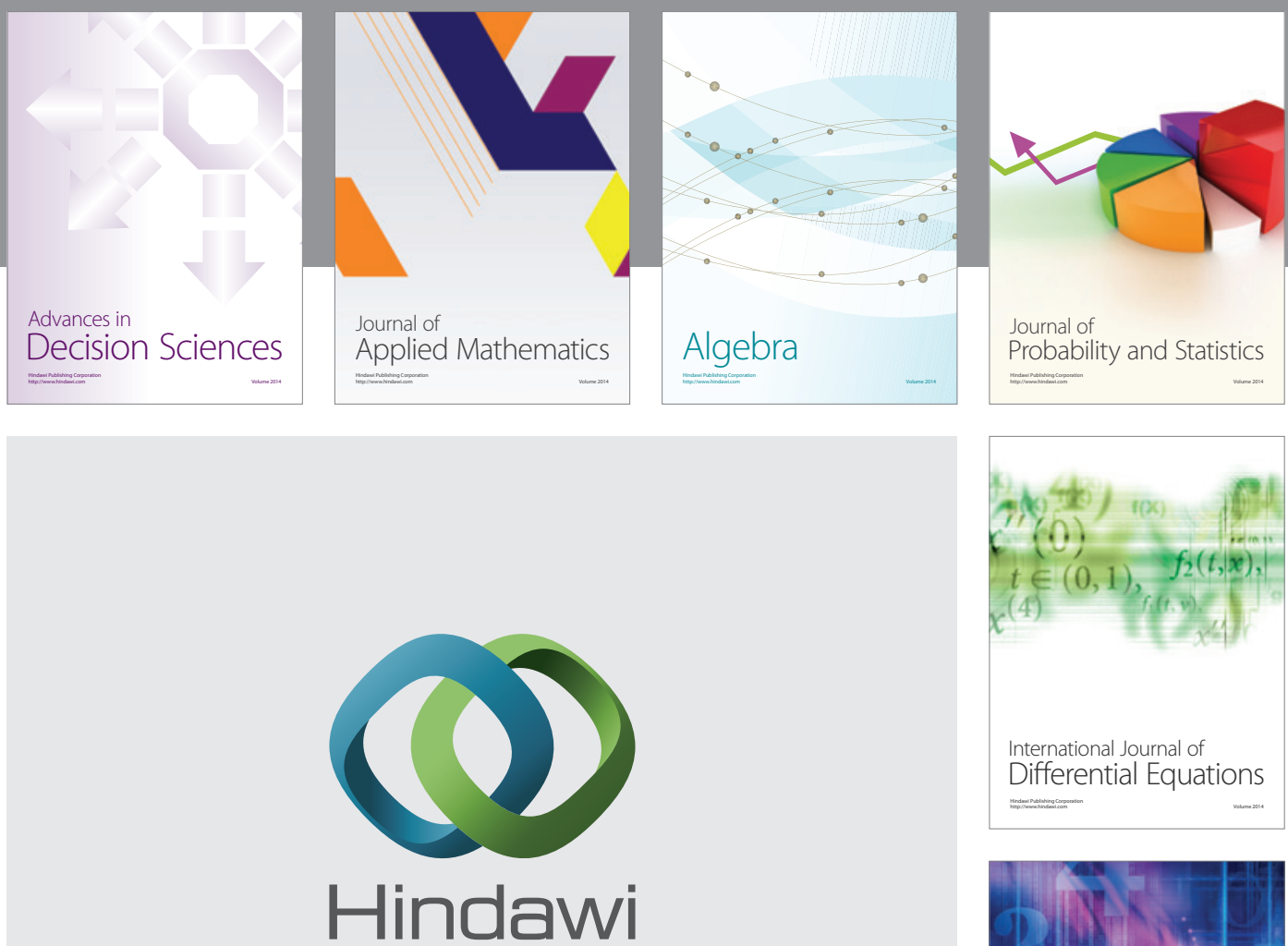

Submit your manuscripts at http://www.hindawi.com
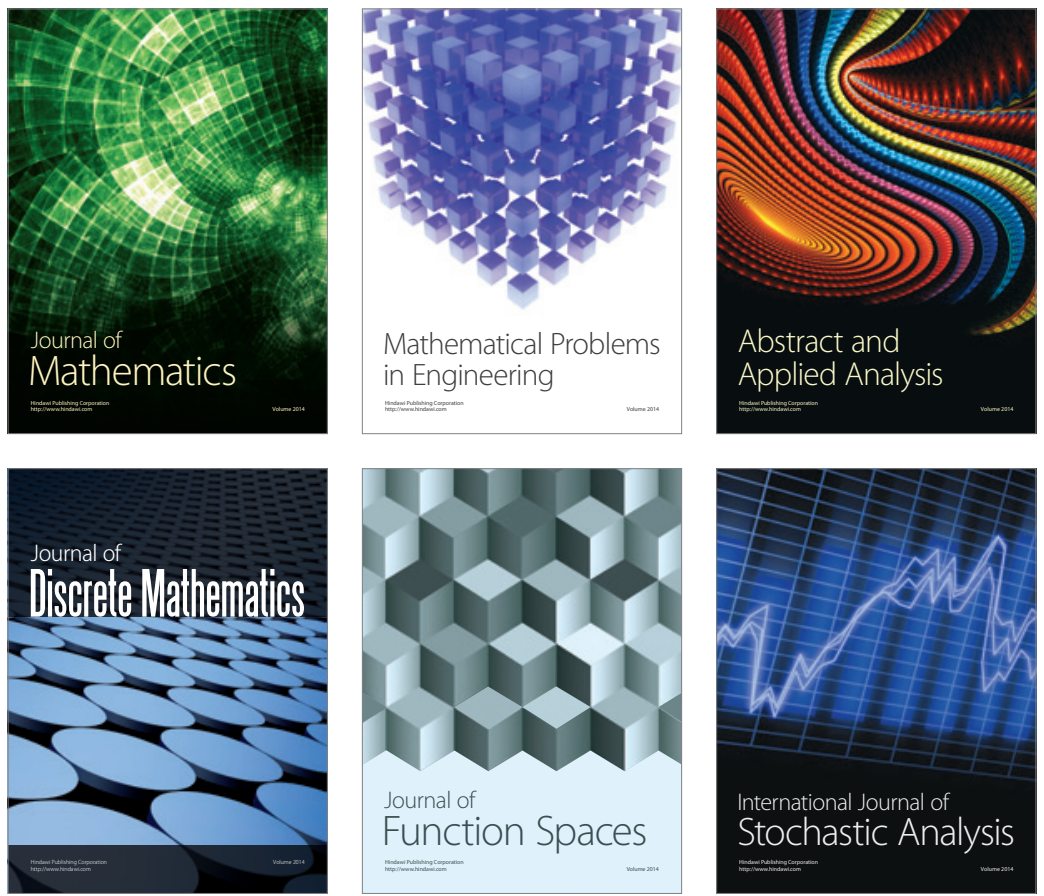

Journal of

Function Spaces

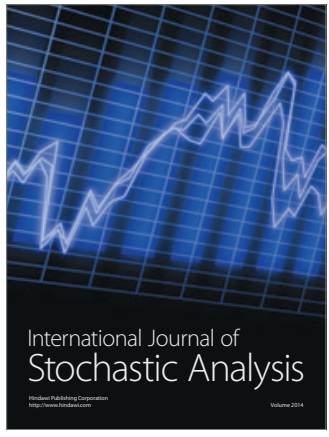

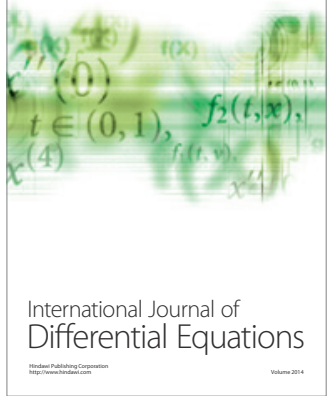
